\title{
RXTE view of the starburst galaxies M 82 and NGC 253
}

\author{
Y. Rephaeli ${ }^{1,2}$ and D. Gruber ${ }^{2}$ \\ 1 School of Physics and Astronomy, Tel Aviv University, Tel Aviv, Israel \\ 2 Center for Astrophysics and Space Sciences, University of California, San Diego, USA
}

Received 25 October 2000 / Accepted 25 March 2002

\begin{abstract}
The two nearby starburst galaxies M 82 and NGC 253 were observed for 100 ksec over a 10-month period in 1997. An increase of the M 82 flux by a factor $\sim 2$ was measured during the period July-November, when compared with the flux measured earlier in 1997. The flux measured in the field centered on M 82 includes $\sim 38 \%$ of the emission from the Seyfert 1 galaxy M 81. The best-fitting model for the earlier emission from M 82 is thermal with $k T \simeq 6.7 \pm 0.1$. In the high flux state, the emission additionally includes either an absorbed second thermal component or absorbed power-law component, with the former providing a much better fit. A likely origin for the temporal variability is a single source in M 82. The flux of NGC 253, which did not vary significantly during the period of observations, can be well fit by either a thermal spectrum with $k T \simeq 3.8 \pm 0.3 \mathrm{keV}$, or by a power law with photon index of $2.7 \pm 0.10$. We have also attempted fitting the measurements to more realistic composite models with thermal and power-law components, such as would be expected from a dominant contribution from binary systems, or Compton scattering of (far) IR radiation by radio emitting electrons. However, the addition of any amount of a power-law component, even with cutoff at $20 \mathrm{keV}$, only increases chi-square. The $90 \%$ confidence upper limit for power law emission with (photon) index 1.5 is only $2.4 \%$ of the $2-10 \mathrm{keV}$ flux of M 82 ; the corresponding limit for NGC 253, with index 2.0, is $48 \%$.
\end{abstract}

Key words. galaxies: starburst - galaxies: individual: M 82, NGC 253 - X-rays: galaxies radiation mechanisms: non-thermal

\section{Introduction}

Interest in X-ray emission in starburst galaxies (SBGs) stems from their diverse radiative activity which is powered by an abundant population of massive, young stars. The enhanced star formation rate in these gas and dust rich galaxies leads to intense far-infrared (FIR) radiation from warm interstellar dust heated by the massive stars. The large number of X-ray binaries and supernova (SN) remnants, as well as hot winds driven by SN shocks, imply that X-ray emission from a SBG is more intense than from a normal spiral (Bookbinder et al. 1980). Moreover, since SN remnants are prime sites for shock-acceleration of particles to high energies, Compton scattering of relativistic electrons by the local radiation fields will enhance X-ray emission in a SBG. This expectation is heightened by the fact that in a SBG the energy density of the FIR radiation field can be much higher than that of the cosmic microwave background radiation (Schaaf et al. 1989; Rephaeli et al. 1991).

The SB phase may occur in various types of galaxies (triggered, perhaps, by galactic mergers). As in other

Send offprint requests to: Y. Rephaeli,

e-mail: yoelr@noga.tau.ac.il active galaxies, a SBG may also have an active, compact nucleus, and one of the primary issues in the study of SBGs is the possible connection between low-luminosity Active Galactic Nuclei (AGN) and SB activities. Since both phenomena may occur in at least some active galaxies, it is important to determine some of their observational manifestations in order to better characterize SBGs. This obviously is a prerequisite in the assessment of the significance of the SB phase in galactic evolution, and its cosmological ramifications, such as the enrichment of the intergalactic medium by metal-rich gas ejected during the SB phase, and the contributions of SBGs to the X-ray background (e.g., Rephaeli et al. 1995).

High energy phenomena are triggered by the massive star formation activity, and because of the obscuration of optical emission and considerable re-processing of IR emission, X-rays allow a more direct and penetrating view of the inner regions of SBGs. Detailed X-ray spatial and spectral information is very valuable for determining the basic properties of SBGs. However, considerable knowledge on the emission mechanisms and environments may be gained from good quality spectral data alone, particularly so if these include temporal information.

M 82 and NGC 253 are the closest SBGs. These "archetypical" SBGs have been observed with all the 
major X-ray satellites (e.g., Fabbiano 1988a; Tsuru et al. 1990; Ohashi et al. 1990; Boller et al. 1992; Matsumoto \& Tsuru 1999; Cappi et al. 1999; Pietsch et al. 2001). Their X-ray emission is quite substantial, in the range $10^{40}-10^{41} \mathrm{erg} / \mathrm{s}$; reported best-fit spectra include both thermal (Cappi et al. 1999) and power-law forms (e.g. Weaver et al. 2000). Both galaxies have already been observed also with Chandra (Strickland et al. 2000; Matsumoto et al. 2001; Kaaret et al. 2001). At low X-ray energies, emission from these galaxies extends well beyond their optical disks, so the SB activity is not manifested only in the central nuclear SB region $(\leq 1 \mathrm{kpc})$. However, even in these nearby galaxies the characteristics of the emission have not yet been determined in much detail. It is important to determine the relative contributions of the SB-powered versus nuclear-powered emission, and the respective significance of thermal and nonthermal processes.

To better establish the X-ray properties of M 82 and NGC 253, we have initiated observations with the Proportional Counter Array (PCA) and the High Energy X-ray Timing Experiment (HEXTE) aboard the Rossi X-ray Timing Explorer (RXTE) satellite. Our main motivation has been to use the good temporal and wide-energy capabilities of the RXTE in order to assess the possible role of an AGN-like variable emission, and the level of contribution of Compton scattering to the integrated emission in SBGs. These RXTE observations and the results of the spectral and temporal analysis are reported here.

\section{Instrumentation and observations}

The RXTE was inserted into low earth orbit on 1995 Dec. 31. The PCA was described by Jahoda et al. (1996) and the HEXTE by Rothschild et al. (1998). The PCA has energy resolution of $16 \%$ at $6 \mathrm{keV}$ and a useful energy range of $2.5 \mathrm{keV}$ to about $25 \mathrm{keV}$, limited at the high end by uncertainties of determining the internal background. The HEXTE has resolution of $15 \%$ at $60 \mathrm{keV}$ and an energy range from 15 to $250 \mathrm{keV}$. For the present observations the errors of background determination with HEXTE were negligible. For both instruments calibration of energy response has been extensive and is accurate to better than $2 \%$. The instrumental time resolution of microseconds was not needed, but the very flexible RXTE scheduling of observations was exploited to permit sampling on all time scales from one orbit $(90 \mathrm{~min})$ to ten months. Both the PCA and HEXTE are non-imaging with field-of-view (FOV) one degree.

Over the period February - November 1997, thirty oneorbit observations each were made of M 82 and NGC 253. To sample possible variability as well as temporal changes through the entire campaign, a somewhat intricate plan (Table 1) was devised consisting of four observing sequences. Eight of the orbits were spaced over one day, seven orbits over one week, another seven over eight weeks, and the remaining eight over 42 weeks. The spacing of observations in each sequence was not uniform but moderately irregular: each sequence was divided into 15 equal segments, and observations were placed in either seven or eight of these segments in patterns based on the cyclic difference set of size 15 (Baumert 1971). Such patterns allow uniform sensitivity to variability on a somewhat broader range of time scales or frequencies than with evenly spaced samples. With the two longest sequences the corresponding M 82 and NGC 253 observations occurred within a day of each other. Three PCA detectors were employed for all observations.

Analysis of M 82 was complicated by variability and by the presence of another known source with appreciable X-ray emission, M 81, in the FOV. The separation of M 81 by $\sim 34^{\prime}$ from $\mathrm{M} 82$ resulted in a transmission of about $38 \%$ for M 81 in the RXTE fields. Although the RXTE field rotated considerably during the 10-month campaign, the PCA FOV, nominally a hexagonal pyramid, is sufficiently conical that the area presented to M 81 varied by not more than $10 \%$. We have also analyzed contemporaneous archival ASCA and ROSAT data to provide limits to the M 81 contribution.

Table 1. Observation sequences.

\begin{tabular}{llll}
\hline \hline Duration & $\begin{array}{l}\text { Frequency } \\
(\mu \mathrm{Hz})\end{array}$ & M 82 Start & NGC 253 Start \\
\hline 1 day & $23.1-81.0$ & 16 July & 13 July \\
1 week & $3.31-11.6$ & 29 March & 11 April \\
8 weeks & $0.41-1.45$ & 28 April & 29 April \\
42 weeks & $0.08-0.28$ & 2 February & 3 February \\
\hline
\end{tabular}

\subsection{Data reduction and background considerations}

The FITS data were reduced with standard FTOOLS into one-orbit light curves and average spectra. Net counting rates for the three PCA detectors averaged $21 \mathrm{~s}^{-1}$ and $0.65 \mathrm{~s}^{-1}$ for $\mathrm{M} 82$ and NGC 253, respectively. The NGC 253 counting rate is not very large compared to the background estimation error of $0.3 \mathrm{~s}^{-1} \mathrm{rms}$ for the three detectors, using the latest weak-source background model for the PCA. An initial display of the PCA spectrum of NGC 253 showed a steep spectrum below $12 \mathrm{keV}$ and above this energy a fairly flat spectrum with weak lines at 25,30 , and $50 \mathrm{keV}$. This upper portion was clearly identifiable with the activation-induced internal background spectrum at about $2 \%$ of the average background level. This spurious component was removed with a $1.9 \%$ correction to the background level. The resulting net spectrum showed no evidence for a statistically significant flux above $12 \mathrm{keV}$ either with PCA or HEXTE. The HEXTE internal background was monitored continuously by beam switching every $16 \mathrm{~s}$ during the observation and required no special treatment.

Field-to-field confusion noise from fluctuations of the diffuse X-ray background (e.g. Shafer 1983) has been calculated (Gruber et al. 1996) to have an rms of $8 \%$ of the background flux, and we calculate this level to be 
$0.4 \mathrm{c} / \mathrm{s}$ for three PCA detectors. This estimated fluctuation level is comparable to the NGC 253 counting rate. The 2-10 keV spectrum of the fluctuations, however, has been shown (Butcher et al. 1997) to have an index of $1.8 \pm 0.02$, which is much flatter than what is observed here for either M 82 or NGC 253. From spectral fitting we determined that the $90 \%$ upper limit to the fraction of the observed $2-10 \mathrm{keV}$ flux which could come from a background fluctuation is $16 \%$ for NGC 253 and less than $1 \%$ for M 82 . Net flux in the FOV of M 82 extends up to $\sim 50 \mathrm{keV}$.

\subsection{Temporal analysis}

We obtained light curves for the M 82 and NGC 253 monitoring observations by fitting each $\sim 3000 \mathrm{~s}$ observation to a Raymond-Smith (R-S) plasma spectral model, then integrating the model flux to obtain $2-10 \mathrm{keV}$ flux. The PCA data do not cover the interval $2-2.5 \mathrm{keV}$, but the 2-10 keV measure should still be quite reliable, given the small gap and lack of noticeable spectral variability.

The thirty NGC 253 one-orbit observations had an average counting rate below $10 \mathrm{keV}$ of $0.65 \pm 0.03 \mathrm{c} / \mathrm{s}$, with reduced chi-square of about unity, and the $\mathrm{rms}$ of $0.16 \mathrm{c} / \mathrm{s}$ is clearly dominated by counting statistics. No variability is evident, and a $90 \%$ limit to random (white) variability at the source is $0.145 \mathrm{c} / \mathrm{s}$, or $22 \%$.

The light curve of M 82, Fig. 1, on the other hand, shows clear evidence for variability on a variety of time scales. Most notably, it appears to be dominated by a single event which doubles the flux late in the monitoring period. We indicate in Fig. 1 the maximum and minimum contributions of M 81 to the observed flux, based on occasional contemporaneous monitoring of M 81 with ASCA (Iyomoto \& Makishima 1999) and ROSAT (Immler \& Wang 2001). The three ASCA and four ROSAT fluxes shown in Fig. 1 were determined from archival data using the standard ftools XSELECT and XSPEC. Obviously, these observed levels do not necessarily sample the full variability of the M 81 flux but clearly they show that a large increase was extremely unlikely. M 82, however, was not monitored with ASCA or ROSAT during the period of RXTE observations. Three ASCA observations just before the RXTE monitoring show both sizeable variability and general consistency with the RXTE fluxes. A BeppoSAX observation of M 82 on December 6-7 1997, about two weeks after the last RXTE observation segment, shows the flux level of the earlier RXTE observations, indicating the end of the elevated flux level. If an example of flaring, this event, with a pattern of fast rise followed by steady emission then fast decay is unusual for X-ray transients, which commonly show a fast rise and slow decay. Abrupt state changes of X-ray emission are seen, however, in the microquasar sources, such as J1655-40 and 1915-05 (Belloni 1998, and references therein), which are generally regarded as black hole candidates. Thus a very interesting possibility is that of an outburst from such
Table 2. Broad-band M 82 variability vs. frequency.

\begin{tabular}{cccc}
\hline \hline Frequency $(\mu \mathrm{Hz})$ & rms & $\sigma$ & $90 \%$ Interval \\
\hline 0.15 & 0.464 & 0.113 & {$[0.303,0.843]$} \\
1.10 & 0.167 & 0.046 & {$[0.101,0.328]$} \\
7.5 & 0.040 & 0.022 & {$[0.000,0.085]$} \\
55. & 0.032 & 0.013 & {$[0.010,0.071]$} \\
\hline
\end{tabular}

a source. Using earlier ASCA data Matsumoto \& Tsuru (1999) and Ptak \& Griffiths (1999) have already inferred the existence of a single strong variable black hole source in M 82. The most obvious explanation, then, is a sudden outburst from a single very luminous source or, much less likely, coincident outbursts from more than one source. One should note, however, that the early data before this event had variability of $10 \%$ rms above counting statistics.

In addition to the single-outburst scenario, we analyzed whether the observed power density spectrum resembles that of known variable sources. In particular, we tested the observed power density for a power-law decline of spectral density with increasing frequency, i.e. a "red" spectrum such as seen in the stellar-size black hole Cyg X-1 (Nolan et al. 1981), in several AGNs (Markowitz \& Edelson 2001), and in HMXRBs generally (Belloni \& Hasinger 1990).

In treating the observed M 82 variability as a red noise process, the measured flux was assumed to be a stochastic variable with power over a broad range of frequencies, increasing with decreasing frequency. Although a finegrained frequency analysis was not possible because of the modest number of samples and marginal statistics, the design of the observing schedule nevertheless permitted a particularly simple analysis: for each of the four sets of observations (specified in the beginning of this section) an average flux for the seven or eight samples was calculated. Values of $\chi^{2}$ were then computed based solely on counting statistics, which resulted in formal values that were unacceptably large, indicating extra variance. Then it was assumed that for each set the flux was intrinsically variable with stationary mean and some variance, or more conveniently - rms. We adjusted this assumed rms and determined the values at which the $\chi^{2}$ probability assumed the values $0.05,0.155,0.5,0.745$ and 0.95 , thus determining the best fit value for the rms and the $68 \%$ and $90 \%$ confidence bounds. The best fit value and standard error for the rms were normalized to the average flux level to obtain a fractional measure of variability. Finally, an average frequency and frequency range were assigned to each variability estimate, thus producing the values in Table 2 and Fig. 2.

The rms clearly decreases strongly with frequency. Fitting to a power-law dependence we obtain an excellent fit with a correlation coefficient of -0.98 and an index of $0.50 \pm 0.02$. This corresponds to an index of unity for the power spectrum, or what is sometimes called a flicker noise spectrum. These observations, then, are consistent with a mildly red noise process extending without flattening to 


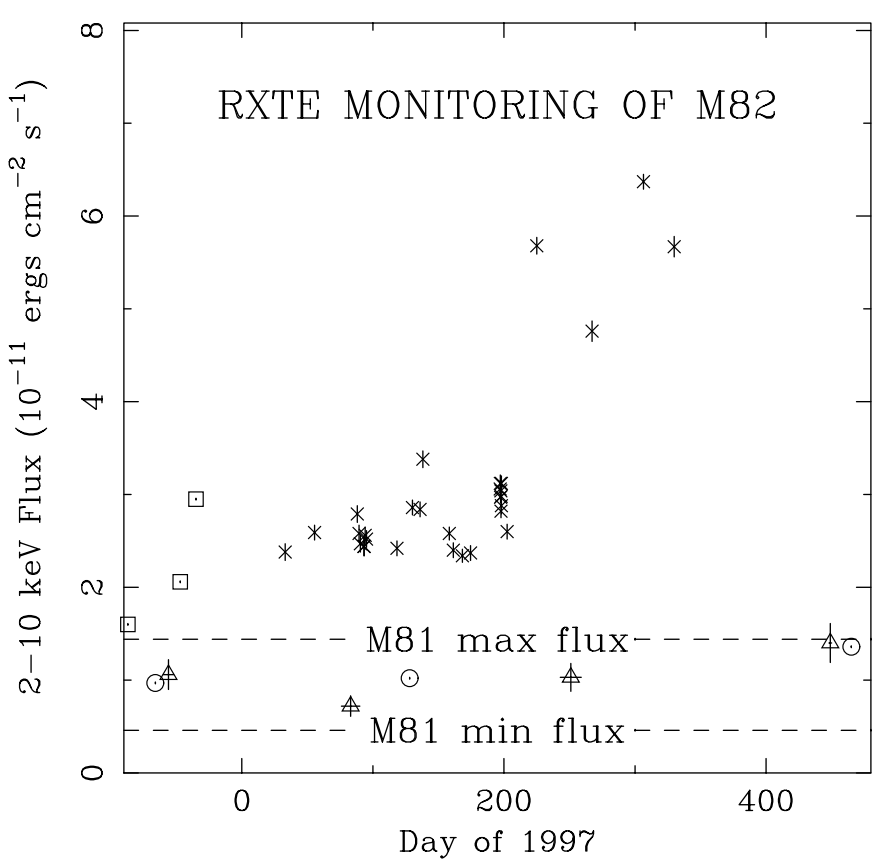

Fig. 1. Observed fluxes of M 82 and M 81 in 1996, 1997 and 1998. RXTE measurements include M 81, which was in the RXTE field at $38 \%$ transmission. The 30 measurements from the RXTE monitoring campaign are marked with x's, ASCA fluxes of M 82 and M 81 are marked with circles and squares, respectively, and the ROSAT fluxes of M 81 are marked with triangles. The observed ROSAT and ASCA fluxes of M 81 have been reduced by the factor 0.38 to correct for the RTE transmission. Reasonable limits to the M 81 contribution, as used in the spectral analysis, are shown by dashed lines.

the lowest frequency of about $0.1 \mu \mathrm{Hz}$. Such a determination contradicts the initial assumption of a stationary process, but not strongly (see Deeter 1984).

Alternatively analyzing the M 82 light curve as having two levels, early (first 26 samples) and late (last 4), we assume that the higher emission at late times represents an addition to the average flux observed at earlier times. We first examine the early emission. Versus time, there is a fitted rise by $20 \%$ from February to July which is formally significant to $3 \sigma$, but has a correlation coefficient of only 0.52 . Therefore we consider the trend to be an incidental effect of more rapid source variability. Assuming a stationary process, we determine a fractional $\mathrm{rms}$ of $0.106 \pm 0.014$, or a $90 \%$ confidence region $[0.083,0.130]$. We estimate the limit to the transition time between the two levels of flux to be about 25 days.

Standard power spectral analysis was used to test whether the observed RXTE $2-10 \mathrm{keV}$ fluxes were more consistent with a step function change in time or with the flicker noise spectrum suggested by Fig. 1. Since the sampling was very uneven, this was done by constructing a comparison time series in which the low and high state data points were each replaced with their respective averages. Power spectra were calculated and the resulting power estimates averaged over four broad bands approximating those in Fig. 1 in order to suppress sampling noise.

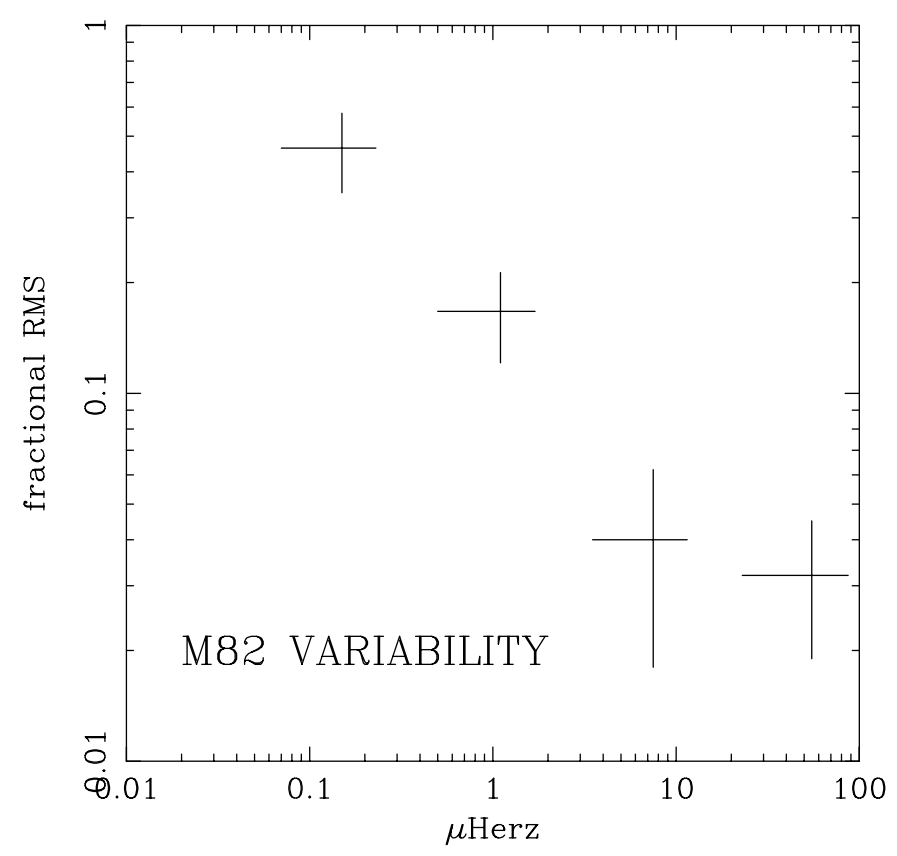

Fig. 2. Power spectrum of flux variability in M 82. This spectrum may indicate a flicker noise process at periods longer than a day.

Within errors of about $25 \%$ per average, the two processes, step change and flicker noise, cannot be distinguished.

We then tried to determine if the four late samples have a pattern of variability distinct from the 26 earlier samples. We find for the last four samples that the fractional rms is 0.098 , about the same as for the early data. Analyzing versus frequency as above, we compute a bestfit index of 0.29 , which is, however, not significantly different from zero $(P=0.19)$ or from $0.5(P=0.30)$. From temporal analysis alone it cannot be determined if the noise process governing the late emission is the same as, or rather different from that of the earlier emission.

In summary, from analysis of the noise properties, we find that the M 82 variability determined from the entire sample is broadly consistent with a power density spectrum of power law form with index 1 . An effort to determine if the late high emission had distinct temporal properties, which could indicate a distinct origin, failed because of the small sample of only four observations.

\subsection{Spectral analysis}

Spectral analysis was done with the use of XSPEC and additional statistical tools. Latest versions of the PCA and HEXTE response matrices were used, and a $\sim 1 \%$ systematic error was assumed in order to allow for errors in the calibration of the PCA response matrix. PCA data above $25 \mathrm{keV}$ were excluded to avoid errors in background modeling. Nevertheless, 3\% negative residuals at $4-5 \mathrm{keV}$, which is the peak of the background spectrum, were attributed to background estimation errors and modeled specially in the fitting. PCA data below $2.5 \mathrm{keV}$ were ignored due to uncertainty of the 
response matrix in the first few channels. These data are thus insensitive to the $0.7 \mathrm{keV}$ thermal component reported by Cappi et al. (1999), Weaver et al. (2000), and Dahlem et al. (2000), and we ignore this component. HEXTE data above $50 \mathrm{keV}$ were felt to add only noise in the fits and were not used for the results reported here.

NGC 253: NGC 253, observed at an average flux level of $2.4 \times 10^{-12} \mathrm{erg} \mathrm{cm}^{-2} \mathrm{~s}^{-1}$ in the $2-10 \mathrm{keV}$ band (or $\sim 0.1$ milliCrab), was significantly detected only by the PCA. Thus HEXTE data were not used for spectral fitting. Since no variability could be determined for this source, data from all thirty $\sim 3000$ s samples were coadded, as well as the background estimates, to produce a mean spectrum. Net detection was at about $20 \sigma$ significance, which was strong enough for simple spectral modeling. We tried power-law and thermal models. For the thermal case we tested the PCA data below $25 \mathrm{keV}$ with both an R-S thermal model and thermal bremsstrahlung with iron line. Both thermal models yielded a temperature of $3.8 \pm 0.3 \mathrm{keV}$. The best-fit Fe abundance in solar units, $Z_{\mathrm{Fe}}$, with R-S was $0.16 \pm 0.08$, and with bremsstrahlung the equivalent width was $160 \pm 130 \mathrm{eV}$. In neither thermal case was an absorbing column required by the data. Values of $\chi^{2}$ are close to 34 for 50 degrees of freedom; although the reduced $\chi^{2}$ is low, it is not sufficiently low to indicate a serious overestimate of errors. An unabsorbed power law model gave a best-fit number index of $2.7 \pm 0.1$, and iron equivalent width of $340 \pm 300 \mathrm{eV}$. (The R-S fit, which tests for several iron lines plus an edge, is more constraining than a fit for a single line.) Although the power law was formally a good fit, with $\chi^{2} \simeq 41.2$ for 50 degrees of freedom, the residuals show correlated departures which indicate that the acceptable $\chi^{2}$ is an artifact of the oversampling in energy. No such correlations are seen in the residuals to the thermal fits. Relative likelihoods from Poisson statistics were calculated from the data and fits, and either thermal form is about 1000 times more likely than the unabsorbed power law. An absorbing column of $N_{\mathrm{H}}=2.8 \times 10^{22} \mathrm{~cm}^{-2}$, however, brings the goodness-of-fit close to the thermal fits. In this case the best-fit index steepens to $3.1 \pm 0.2$. The fitting was performed on the interval $2-22 \mathrm{keV}$, which includes many channels above $12 \mathrm{keV}$, where the source is no longer visible. However, the best fit parameters and their errors are very insensitive to a variation of the upper cutoff energy, even when it is lowered to $10 \mathrm{keV}$.

Because of the faintness of NGC 253, the best-fit spectral parameters show a moderate dependence on the background subtraction. The value of $\chi^{2}$ changes significantly when the background level is adjusted by $\pm 0.5 \%$, for which we obtain corresponding changes for best-fit temperature of $0.7 \mathrm{keV}, Z_{\mathrm{Fe}} \simeq 0.05$ for abundance, and 0.15 for spectral index. These systematic errors resulting from the uncertainty of background subtraction are less than twice the formal statistical errors from counting statistics. Somewhat smaller errors can be associated with a fluctuation of the X-ray background. At the 90\% upper limit of $16 \%$ of the net $2-10 \mathrm{keV}$ flux attributable to such a fluctuation, the best-fit temperature is then smaller by $0.4 \mathrm{keV}$ and $Z_{\mathrm{Fe}}$ is larger by 0.03 .

M 82: Spectral analysis of M 82 includes consideration of the maximum and minimum possible contribution from the confusing source M81. From these limiting cases we show that the spectral analysis is not seriously compromised by M 81. It was particularly interesting to observe the difference between the late high-flux emission and the earlier low emission (Fig. 1), with no allowance being made yet for any contribution from M 81. This lower flux averaged $2.9 \times 10^{-11}$ erg $\mathrm{cm}^{-2} \mathrm{~s}^{-1}$ in the $2-10 \mathrm{keV}$ band, or $\sim 1.3$ milliCrab, and the average for the four high samples was almost exactly double this level. We analyzed spectra summed over the whole campaign, the 26 (low) earlier observations, the last 4 (high) observations, and finally obtained a difference spectrum. The early, late and difference spectra all show an overwhelming preference for an exponential shape over an unabsorbed power law - the reduced $\chi^{2}$ using the power law form lies in the range $3-4$. Even a small power-law component results quickly in significantly increased $\chi^{2}$ : a $15 \%$ contribution increases $\chi^{2}$ by 12 in all but the low-flux spectra. A comparison of Tables 3-5 below shows the rapid worsening of $\chi^{2}$ with increasing power law fraction as represented by the powerlaw M 81 contribution.

Results for the best-fit thermal unabsorbed and absorbed models for the total emission in the FOV of M 82, i.e. without allowance for an M81 contribution, are presented in Table 3. To represent thermal emission we employed both a Raymond-Smith plasma model and a thin thermal bremsstrahlung with a separate single iron line. Empirically, we find little difference between the two forms: values of $\chi^{2}$ were almost identical, as were the bestfit values of $k T$, and the iron line centroid floated to values near $6.7 \mathrm{keV}$. In the "quiescent" low-brightness phase, $k T=7.35 \pm 0.07 \mathrm{keV}$, and the Fe line strength is represented by an equivalent width of $120 \pm 30 \mathrm{eV}$, or with the R-S model by $Z_{\mathrm{Fe}}=0.10 \pm 0.01$. The "outburst", or difference emission (high-low) is best-fit by an absorbed thermal spectrum with $k T=4.72 \pm 0.17 \mathrm{keV}$, but has a very weak iron line of equivalent width $50 \pm 70 \mathrm{eV}$, or in the R-S model $Z_{\mathrm{Fe}}=0.02 \pm 0.02$.

The above analysis was then repeated assuming maximal contribution from M81, given its variability. The long term 2-10 keV flux of M81 (as measured by six $\mathrm{X}$-ray satellites) seems to have varied by a factor of $\sim 3$ during the last twenty years (see Fig. 2 in Pellegrini et al. 2000). In 1997, when the RXTE observations were made, ASCA measured a flux which varied in the range $\sim(2.4-3.4) \times 10^{-11}$ erg $\mathrm{cm}^{-2} \mathrm{~s}^{-1}$ in this energy band (Iyomoto \& Makishima 1999). This is also the largest variation observed over a single year. A slightly higher flux, $3.8 \times 10^{-11} \mathrm{erg} \mathrm{cm}^{-2} \mathrm{~s}^{-1}$, was measured by BeppoSAX in June 1998 (Pellegrini et al. 2000); their best-fit continuum spectrum was determined to be a power-law with photon index $\alpha=1.86$. The flux range measured by 
Table 3. Summary of thermal spectral fits to the full emission in the FOV of M 82.

\begin{tabular}{lcccccc}
\hline \hline Dataset & $\begin{array}{c}\chi^{2} \\
(110 \mathrm{df})\end{array}$ & $\begin{array}{c}N_{\mathrm{H} \mathrm{cm}^{-2 a}} \\
\left(10^{22} \mathrm{~cm}^{-2}\right)\end{array}$ & $\begin{array}{c}k T^{a} \\
(\mathrm{keV})\end{array}$ & $\begin{array}{c}\text { Abundance } \\
(\text { solar })\end{array}$ & $\begin{array}{c}\text { Fe Eq. Width } \\
(\mathrm{eV})\end{array}$ & Flux $^{b}$ \\
\hline All & 139 & 0 & $7.08 \pm 0.08$ & $0.105 \pm 0.007$ & $130 \pm 20$ & 3.36 \\
Low & 113 & 0 & $7.35 \pm 0.07$ & $0.102 \pm 0.009$ & $120 \pm 30$ & 2.95 \\
High & 140 & 0 & $6.59 \pm 0.09$ & $0.103 \pm 0.012$ & $150 \pm 30$ & 5.80 \\
High-Low & 140 & 0 & $5.91 \pm 0.16$ & $0.097 \pm 0.023$ & $170 \pm 70$ & 3.36 \\
High-Low & 98 & $2.7 \pm 0.4$ & $4.72 \pm 0.17$ & $0.020 \pm 0.020$ & $50 \pm 70$ & 3.93 \\
\hline
\end{tabular}

${ }^{a}$ All quoted errors are at the $68 \%$ confidence level.

${ }^{b}$ Flux in $2-10 \mathrm{keV}$ band, in units of $10^{-11} \mathrm{erg}^{-2} \mathrm{~cm}^{-1}$.

ASCA implies - with the $38 \%$ transmission - that the M 81 emission constitutes $\sim 31 \%-44 \%$ of the counting rate in the low samples, and $\sim 16 \%-22 \%$ of the high average. The results of repeating the fits of Table 3 in the presence of the highest ASCA-measured flux from M 81, assuming a power-law spectrum with index 1.8, are collected in Table 4. A comparison of Tables 3 and 4 indicates that the best-fit spectral parameters do not depend strongly on the uncertain contribution of M 81. The bestfit thermal R-S model (Fig. 3) to the low-state emission has $k T$ reduced by $10 \%$ from $7.3 \mathrm{keV}$ (without explicit accounting for emission from M81) to 6.7 (with maximal M 81 flux), and has a somewhat higher value for $\mathrm{Fe}$ abundance, $Z_{\mathrm{Fe}}=0.14 \pm 0.01$. This value of the temperature is appreciably lower than the best-fit value deduced from the BeppoSAX measurements of Cappi et al. (1999), $k T=8.20 \pm 0.59 \mathrm{keV}$; their value for the $Z_{\mathrm{Fe}}, 0.07 \pm 0.02$, is very low and formally inconsistent with these RXTE results.

We tried also a composite thermal plus power law spectrum to simulate an average Galactic binary X-ray spectrum (White et al. 1983), which has recently been used to model the contribution of binaries to X-ray emission in SBGs (Persic \& Rephaeli 2002). A full representation of such a model requires specifying various components with a range of parameter values (including cutoff energies), most of which are poorly known. Clearly, only simple spectral modeling is warranted with our RXTE measurements; indeed, even with the more constraining spatiallyresolved spectral analysis afforded by the joint ROSAT and ASCA datasets, only a simple combination of thermal plus power-law components was used to characterize the integrated emission of massive binary systems in SBGs (Dahlem et al. 1998; Dahlem et al. 2000; Weaver et al. 2000). Adding a cut-off power-law spectrum with a photon index of 1.5 (Dahlem et al. 1998) we found that any amount of such emission increased $\chi^{2}$ from the pure thermal case, and we obtained a $90 \%$ confidence limit of $2.4 \%$ of $2-10 \mathrm{keV}$ flux in this component. This result is not very sensitive to the assumed level of M81 emission.

We consider also absorbed thermal and power law spectral forms for the extra emission in the late four samples (Tables $3-5$ ). The only acceptable $\chi^{2}$ was obtained with an absorbed thermal model (Fig. 4)

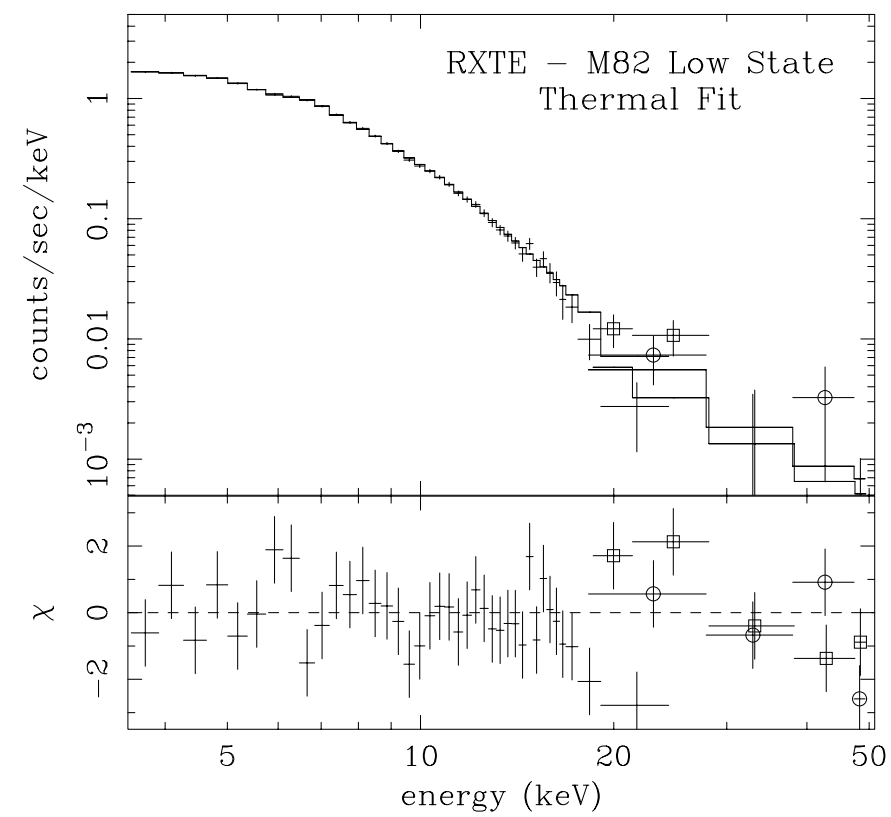

Fig. 3. Low-state M 82 emission and best-fit R-S model with $6.66 \pm 0.08 \mathrm{keV}$. HEXTE data are marked with circles (cluster A) and squares (cluster B). The M 81 contribution is set at the maximum of Fig. 1. The lower panel shows acceptable residuals to the fit.

with $k T=3.80 \pm 0.15 \mathrm{keV}, \quad Z_{\mathrm{Fe}}=0.04$, and column $N_{\mathrm{H}}=4.3 \times 10^{22} \mathrm{~cm}^{-2}$. The best-fit power law to the residual emission was steep, index greater than 3 . Qualitatively similar results were obtained whether the fits were performed after subtraction of the lower M 81 ASCA flux, or the higher BeppoSAX fluxes. As can be seen in the middle panel of Fig. 4, residuals to a fit which neglects absorption leave a pattern of correlated residuals over the entire span of 3-20 keV.

The best-fit thermal forms for the late extra emission have temperatures that are modestly but significantly lower than for the earlier emission. If the entire data set samples a single source and process, then this process must generate somewhat softer spectra at higher emission levels. We note that whether the extra emission is assumed to have a thermal or a power law form, the addition of an absorbing column results in a significant improvement in $\chi^{2}$, dramatically so for the power law form. The appearance 
Table 4. Results of thermal spectral fits for M 82, with high M 81 component.

\begin{tabular}{lcccccc}
\hline \hline Dataset & $\begin{array}{c}\chi^{2} \\
(110 \mathrm{df})\end{array}$ & $\begin{array}{c}N_{\mathrm{H}} \\
\left(10^{22} \mathrm{~cm}^{-2}\right)\end{array}$ & $\begin{array}{c}k T \\
(\mathrm{keV})\end{array}$ & $\begin{array}{c}\text { Abundance } \\
(\text { solar })\end{array}$ & $\begin{array}{c}\text { Fe Eq. Width } \\
(\mathrm{keV})\end{array}$ & Flux $^{a}$ \\
\hline 3.40 & & & & & & \\
All & 175 & 0 & $6.46 \pm 0.06$ & $0.138 \pm 0.008$ & $180 \pm 30$ & 1.92 \\
Low & 137 & 0 & $6.66 \pm 0.08$ & $0.141 \pm 0.010$ & $190 \pm 60$ & 1.55 \\
High & 153 & 0 & $6.22 \pm 0.09$ & $0.120 \pm 0.012$ & $200 \pm 40$ & 4.40 \\
High-Low & 159 & 0 & $5.17 \pm 0.16$ & $0.147 \pm 0.028$ & $220 \pm 80$ & 2.89 \\
High-Low & 100 & $4.3 \pm 0.5$ & $3.80 \pm 0.15$ & $0.037 \pm 0.022$ & $110 \pm 120$ & 3.76 \\
\hline
\end{tabular}

See footnotes to Table 3 .

Table 5. Power law spectral fits to the high state increment.

\begin{tabular}{lccccc}
\hline \hline M 81 Emission & $\begin{array}{c}\chi^{2} \\
(110 \mathrm{df})\end{array}$ & $\begin{array}{c}N_{\mathrm{H}} \\
10^{22} \mathrm{~cm}^{-2}\end{array}$ & Index & $\begin{array}{c}\text { Fe Eq. Width } \\
(\mathrm{eV})\end{array}$ & Flux $^{a}$ \\
\hline Not included & 292 & 0 & $2.38 \pm 0.02$ & $310 \pm 180$ & 2.89 \\
Not included & 123 & $6.8 \pm 0.6$ & $3.02 \pm 0.06$ & $130 \pm 30$ & 5.23 \\
Included & 307 & 0 & $2.45 \pm 0.03$ & $390 \pm 90$ & 2.38 \\
Included & 133 & $8.6 \pm 0.8$ & $3.32 \pm 0.08$ & $170 \pm 90$ & 5.19 \\
\hline
\end{tabular}

${ }^{a}$ Intrinsic, unabsorbed $2-10 \mathrm{keV}$ power law flux in units of $10^{-11} \mathrm{erg} \mathrm{cm}^{-2} \mathrm{~s}^{-1}$.

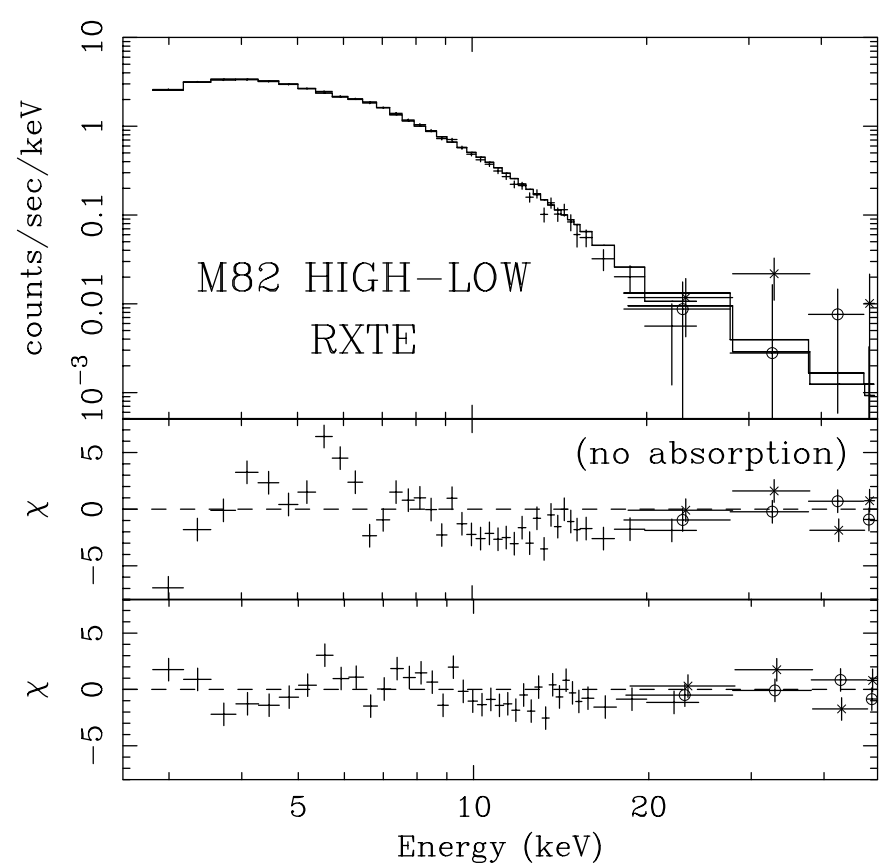

Fig. 4. The extra emission in the high flux state of M 82 and best-fit absorbed R-S model with $k T=3.80 \pm 0.15 \mathrm{keV}$. HEXTE data are marked as in Fig. 3. The lowest panel shows the acceptable residuals to the best-fitting absorbed extra emission, while the middle panel shows a broad pattern of correlated and unacceptable residuals to an unabsorbed extra component.

of an extra column with increased flux levels may not be unphysical even if the high and low levels result from a single process, but it is simpler to interpret the need for absorption as an indication of an obscured source. Thus, based on this spectral analysis it would appear that the origin of the extra late emission is in a source, most likely a single source, which did not emit the earlier lower flux.

A similar high-low analysis has been performed by Matsumoto \& Tsuru (1999) and by Ptak \& Griffiths (1999), both using essentially the same ASCA data collected from 1993 to 1996. Matsumoto \& Tsuru give the more useful summary of spectral fitting of the high-low flux: a thermal form with best-fit $k T=11.7 \pm 2.5 \mathrm{keV}$, fits slightly better than a power law with best-fit number index 1.8. Both forms require strong absorption with column near $1.4 \times 10^{22} \mathrm{~cm}^{-2}$. These RXTE results, with much better high energy sensitivity, strongly favor a thermal form over power law, but the best fit values for column and $k T$, $2.7 \times 10^{22}$ and $4.7 \mathrm{keV}$, respectively, each differ from the corresponding ASCA value by a factor of two. Differences of this scale probably cannot be dismissed as intercalibration errors: ASCA and RXTE saw something rather different. If the same single source was seen, it varies on the scale of roughly a year in both column and general spectral shape. Much of the absorbing column must lie near to the source.

\section{Discussion}

Given the varied nature of X-ray emitting environments in SBGs, significant thermal emission is clearly expected from the relatively high abundance of various stellar sources such as X-ray binaries, hot gas in SNRs, and SNdriven winds in the IS space and inner halo. The main 
issues that can be addressed based directly on our spectral analysis have been briefly discussed in the previous section. Here we elaborate further on the implications of these results.

A detailed comparison between the results of the RXTE and BeppoSAX observation is complicated, given that the PCA has a larger FOV than that of the MECS experiment. Also, no temporal comparison is possible because the observations were made over non-overlapping periods, and due to the fact that BeppoSAX observed M 82 over only two days. It is nonetheless of considerable interest that BeppoSAX measurements do show some flux variability in the central region of M 82 . This is a clear indication that at least part of the variability seen in the PCA data is intrinsic to M 82, and is not fully attributable to the variable (Seyfert 1) galaxy M 81, which is in the FOV of the PCA but not in that of the MECS. Some, seemingly periodic, variability of the M 81 emission on a timescale of up to $\sim 2$ days, has been seen recently by BeppoSAX (Pellegrini et al. 2000). If this is characteristic of M 81, then it is clear that the stronger variability detected by the RXTE over a longer timescale has a different origin. We also note - from this general comparative point of view - that there is no indication of a second, lowtemperature component in the RXTE data, whose lower threshold energy at $\sim 3.5 \mathrm{keV}$ makes RXTE insensitive to it.

An important result of our analysis is the large secular variation in the flux of M 82 during the last four observation segments in July 1997. Such a large temporal change of galactic-scale emission is commonly attributed to the characteristics of the accretion process onto a massive black hole. These include also power law noise spectra which have been observed for several AGN sources (e.g., Edelson \& Nandra 1999) with spectral indices of 1.5-1.7, only mildly inconsistent with the present case of $1.0 \pm 0.4$. The variable emission would then be expected to have an AGN-like power law energy spectrum with very significant photoelectric absorption. Indeed, our spectral analysis yields evidence for the presence of such a component in the variable emission from M 82. The deduced values of $N_{\mathrm{H}}$ are rather high, $\sim(3-20) \times 10^{22} \mathrm{~cm}^{-2}$ (about an order of magnitude higher than column densities in Seyfert I galaxies such as M81), perhaps indicative of a Seyfert II nucleus (e.g., Risaliti et al. 1999). However, the best-fit power law index, $\alpha \sim 3.9$, is much higher than the more typical value of $\sim 1.8$. The thermal fit of the residual emission with the high absorption yields a very low iron abundance, perhaps too low to be acceptable.

Alternatively, the flux increase in July 1997 may possibly be due to variable emission from an X-ray binary system. If in M82, the high level of emission $(L \geq$ $4 \times 10^{40} \mathrm{erg}^{-\mathrm{s}^{-1}}$ in the $2-10 \mathrm{keV}$ band) would make this unusually luminous when compared with the more common luminosity range $\left(10^{36}-10^{38} \mathrm{erg} \mathrm{s}^{-1}\right)$ of binaries. Although rare, a few such X-ray luminous binaries are known (e.g., in the spiral galaxies NGC 3628 and NGC 4631 - Fabbiano 1988b). The spectral shapes of these sources are usually fit with power-law indices $<2$, or $k T$ values of a few $\mathrm{keV}$. Arguing against a binary origin for the extra emission is the very low upper limit of $15 \mathrm{eV}$ for the equivalent width of an $\mathrm{Fe} \mathrm{K} \alpha$ line. The $320 \mathrm{eV}$ equivalent width of Hercules X-1 Fe K $\alpha$ line (Gruber et al. 2001) reasonably typical of Galactic accreting binaries, is a factor $\sim 20$ higher.

From ASCA monitoring in 1993 and 1999 it was concluded (Matsumoto \& Tsuru 1999; Ptak \& Griffiths 1999) that the M 82 flux varied by a factor $\sim 4$ over this period. They argue that the varying emission originates from within a central $10^{\prime \prime}$ region, and that if due to accretion onto a massive black hole, the implied mass is $\geq 460 M_{\odot}$. A compact source in the nucleus with the deduced level of luminosity, $\sim 4.9 \times 10^{40} \mathrm{erg} / \mathrm{s}$ in the $2-10 \mathrm{keV}$ band (based on a distance of $3.6 \mathrm{Mpc}$ ), would imply that M 82 is a low luminosity AGN. However, we consider the identification of the source of flux variability with an AGN in M 82 to be insecure. Continued monitoring of the central emission in M 82 is needed in order to better establish its temporal, spectral, and spatial properties. More recently, highresolution observations of the central $1^{\prime}$ region of M 82 with the Chandra satellite revealed 4 sources whose flux exhibits significant temporal variability (Matsumoto et al. 2001; Kaaret et al. 2001). In particular, the $0.5-10 \mathrm{keV}$ flux of one of these sources (CXOM82 J095550.2+694047) varied by a factor of $\sim 7.2$ (during the period October 1999January 2000). Matsumoto et al. (2001) suggest that this source is the origin of the variability detected by ASCA. In its high flux state, the emission from this source is comparable to the high-low flux we deduce from the RXTE measurements.

Irrespective of the origin of the variable spectral component in M 82, it is important to note that about half of the measured emission is at most weakly varying. Even though the superposed emission from many variable sources is non-varying, given the thermal (unabsorbed) character of the non-varying emission, and previous evidence for its spatial extent, it is reasonable to conclude that this emission is powered by starburst activity.

Cappi et al. (1999) have suggested that the main hightemperature spectral components, which account for most of the observed 2-10 keV emission in both NGC 253 and M 82, are largely due to emission from hot galactic winds. While we do expect the intense star formation activity to drive hot galactic winds, it is clear that thermal emission in SBGs is a superposition of emissions from a population of X-ray binaries, SN remnants, and galactic winds. The determination of the exact origin of the main high-temperature spectral component will be possible only when more sensitive spectral and imaging measurements are made of NGC 253 and M 82.

Both BeppoSAX and RXTE find no evidence for variability in the emission from NGC 253, so this galaxy is not dominated by an AGN. The best-fit temperature derived from the BeppoSAX measurements (Cappi et al. 1999), $\simeq 5.75 \pm 0.52 \mathrm{keV}$ (90\% confidence), is roughly consistent with our best-fit value of $4.2 \pm 0.4$ ( $68 \%$ confidence), when 
the uncertainty in the background subtraction is included. Even though the value of the iron abundance we determine here is lower than that deduced from the BeppoSAX measurements, both are substantially uncertain and (therefore) in rough agreement.

Power law and thermal models were previously found to provide acceptable fits to the emission observed by ASCA from both M 82 \& NGC 253 (Ptak et al. 1997.) In fact, Moran \& Lehnert (1997), who analyzed 1993 ASCA observations, concluded that the main component of the emission in M 82 is nonthermal, and based on the similar values of the X-ray and radio power law indices, they suggested that the emission is from Compton scattering of relativistic electrons by the local radiation fields. Indeed, the low iron abundances we deduce here are puzzling given the intense star formation activity, and the associated processing of metal enriched interstellar gas in these galaxies. Higher abundances are obtained not only when the dominant spectral component of the continuum emission is a power law, but in any case where nonthermal emission contributes appreciably to the overall emission. For example, if the $2-10 \mathrm{keV}$ flux in the power law component is taken to be $25 \%$ of the thermal component, then the iron abundance in M 82 increases from its best-fit value 0.14 (Table 4) to 0.23 .

It has previously been shown by Goldshmidt \& Rephaeli (1995) that Compton scattering of radio producing relativistic electrons by the far infrared radiation field can account for the substantial high energy (50$150 \mathrm{keV}$ ) emission detected by OSSE (aboard CGRO) from NGC 253 (Bhattacharya et al. 1994). The similarities in radio properties of M 82 and NGC 253 and their common starburst nature are sufficiently strong indications that Compton scattering may play an appreciable role also in M 82. However, neither the BeppoSAX PDS nor the HEXTE observations were sufficiently sensitive to clearly detect this component at high energies where it can dominate the emission.

Acknowledgements. We acknowledge useful comments by the referee on an earlier version of the paper. This work was supported by NASA Grant NAS5-4623 at UCSD. Archival data analysis reported in this paper was aided by tools provided by NASA/HEASARC.

\section{References}

Baumert, L. D. 1971, Lect. Notes Math., v. 182

Belloni, T. 1998, New Astr. Rev., 42, 585

Belloni, T., \& Hasinger, G. 1990, A\&A, 230, 103

Bhattacharya, D., The, L.-S., Kurfess, J. D., et al. 1994, ApJ, 437, 173

Boller, Th., Meurs, E. J. A., Brinkmann, W., et al. 1992, A\&A, 261,57
Bookbinder, J., Cowie, L. L., Krolik, J. H., et al. 1980, ApJ, 237, 647

Butcher, J. A., Stewart, G. C., Warwick, R. S., et al. 1997, MNRAS, 291, 437

Cappi, M., Persic, M., Bassani, L., et al. 1999, A\&A, 350, 777

Dahlem, M., Weaver, K. A., \& Heckman, T. M. 1998, ApJS, 118, 401

Dahlem, M., Parmar, A., Oosterbroek, T., et al. 2000, ApJ, 538,555

Deeter, J. E. 1984, ApJ, 261, 337

Edelson, R., \& Nandra, K. 1999, ApJ, 514, 682

Fabbiano, G. 1988a, ApJ, 330, 672

Fabbiano, G. 1988b, ApJ, 324, 749

Goldshmidt, O., \& Rephaeli, Y. 1995, ApJ, 444, 113

Gruber, D. E., Blanco, P. R., Heindl, W. A., et al. 1996, A\&AS, 120,641

Gruber, D. E., Heindl, W. A., Rothschild, R. E., et al. 2001, ApJ, 562, 499

Immler, S., \& Wang, Q. D. 2001, ApJ, 554, 202

Iyomoto, N., \& Makishima, K. 1999, Astron. Nachr., 320, 300I

Jahoda, K., Swank, J. H., Giles, A. B., et al. 1996, Proc. SPIE, 2808, 59

Kaaret, P., Prestwich, A. H., Zezas, A., et al. 2001, MNRAS, 321, L29

Markowitz, A., \& Edelson, R. 2001, ApJ, 547, 684

Matsumoto, H., \& Tsuru, T. G. 1999, PASJ, 51, 321

Matsumoto, H., Tsuru, T. G., Koyama, K., et al. 2001, ApJ, 547, L25

Moran, E. C., \& Lehnert, M. D. 1997, ApJ, 478, 172

Nolan, P. L., Gruber, D. E., Matteson, J. L., et al. 1981, ApJ, 246,494

Ohashi, T., Makishima, K., Tsuru, T., et al. 1990, ApJ, 365, 180

Pellegrini, S., Cappi, M., Bassani, L., et al. 2000, A\&A, 353, 447

Persic, M., Mariani, S., Cappi, M., et al. 1998, A\&A, 339, L33

Persic, M., \& Rephaeli, Y. 2002, A\&A, 382, 843

Pietsch, W., Roberts, T. P., Sako, M., et al. 2001, A\&A, 365, L174

Ptak, A., \& Griffiths, R. 1999, ApJ, 517, L85

Ptak, A., Serlemitsos, P., Yaqoob, T., et al. 1997, AJ, 113, 1286

Rephaeli, Y., Gruber, D., MacDonald, D., \& Persic, M. 1991, ApJ, 380, L59

Rephaeli, Y., Gruber, D., \& Persic, M. 1995, A\&A, 300, 91

Risaliti, G., Maiolino, R., \& Salvati, M. 1999, ApJ, 522, 157

Rothschild, R. E., et al. 1998, Space. Sci. Instr., 4, 265

Schaaf, R., Pietsch, W., Biermann, P. L., Kronberg, P. P., \& Schmutzler, T. 1989, ApJ, 336, 722

Shafer, R. 1983, Ph.D. Dissertation, University of Maryland

Strickland, D. K., Heckman, T. M., Weaver, K. A., \& Dahlem, M. 2000, AJ, 120, 2965

Tsuru, T., Ohashi, T., Makishima, K., Mihara, T., \& Kondo, H. 1990, Publ. Astron. Soc. Jpn., 42, L75

Weaver, K. A., Heckman, T. M., \& Dahlem, M. 2000, ApJ, 534,684

White, N. E., Swank, J. H., \& Holt, S. S. 1983, ApJ, 270, 711 